\title{
SELECTIVE AND LOCALIZED BONDING USING INDUCTION HEATING
}

\author{
Andrew Cao, Mu Chiao \& Liwei Lin \\ Department of Mechanical Engineering \\ University of California at Berkeley \\ Berkeley Sensor And Actuator Center \\ Berkeley, CA, 94720,acao@me.berkeleyedu
}

\begin{abstract}
Selective and localized bonding using induction heating has been successfully demonstrated for MEMS (Microelectromechanical Systems) packaging and fabrication applications. Eddy current is generated when a closed metal bonding ring is placed under an alternating magnetic field. The metal bonding rings function both as the heating source and bonding solder. Both Pyrex-to-Pyrex and Pyrex-to-polycarbonate bonding processes have been achieved. The heating cycle needed to complete the bonding process is $100 \mathrm{~ms}$ and 60 seconds for the polymer and Pyrex bonding processes respectively. The low thermal budget allows water to be encapsulated inside a cavity without evaporating. Experimentally, Pyrex substrates can be hermetically bonded using gold bonding rings. The gold bonding ring can be used as a solder that bonds the substrates, or it can be used as a heat source to locally meld the glass and facilitates direct Pyrex-to-Pyrex bonding. In either case, the bond was very strong and managed to maintain a hermetically sealed cavity.
\end{abstract}

\section{INTRODUCTION}

Hermetic sealing and packaging is vital in MEMS device applications for long-term stability. It is well known that hermetic packaging in the chip level is expensive and can contribute to a major portion of the overall cost of a device. Wafer-level packaging can substantially reduce the price of packaging and overall manufacturing cost. Therefore, many researchers have worked on various approaches for hermetical sealing processes either with or without enclosed MEMS devices. For example, an LPCVD (Low Pressure Chemical Vapor Deposition) silicon nitride sealing process has been used to encapsulate micro-resonators in vacuum environment [1,2]. A resistive joule heating method has been used to seal sensors and resonators in hermetic and vacuum environments [3-5]. A RTP (Rapid Thermal Processing) bonding process has been applied to MEMS packaging for hermetic and vacuum applications [6-7]. The introduction of selective and localized bonding using induction heating takes the advantages of the above processes, including localized and selective bonding. Induction heating is suitable for parallel processing and it is relatively easy to implement. As such, this new class of bonding method may have potential for MEMS packaging applications.

Induction heating is commonly used in industry for applications ranging from welding, heat treatment of metals and bottle sealing. In microelectronics processing, induction heating has been proposed for whole-wafer bonding process [8]. Previously, induction heating has been demonstrated to remotely and selectively applied heat to micro bonding rings [9]. This paper uses induction heating to perform hermetic bonding of glass wafers and plastic wafers and demonstrates the feasibility of encapsulating liquid.

\section{THEORY}

Selective and localized induction heating is based on Faraday's law. Electric field and eddy current are generated on metal rings by placing the ring inside a changing magnetic field.
Joule heating is generated remotely and selectively on localized areas. Figure 1 shows the schematic diagram of this concept. With proper design and process control, the heating area can be confined selectively and locally for the purpose of bonding applications. In the prototype demonstration, a cavity drilled in the center of the bonding ring is shown in Fig. 1, and is filled with water to test the feasibility of localized heating without vaporizing the water. If the heating causes the water to evaporate, the steam created would interfere with the bonding process, making encapsulation impossible.

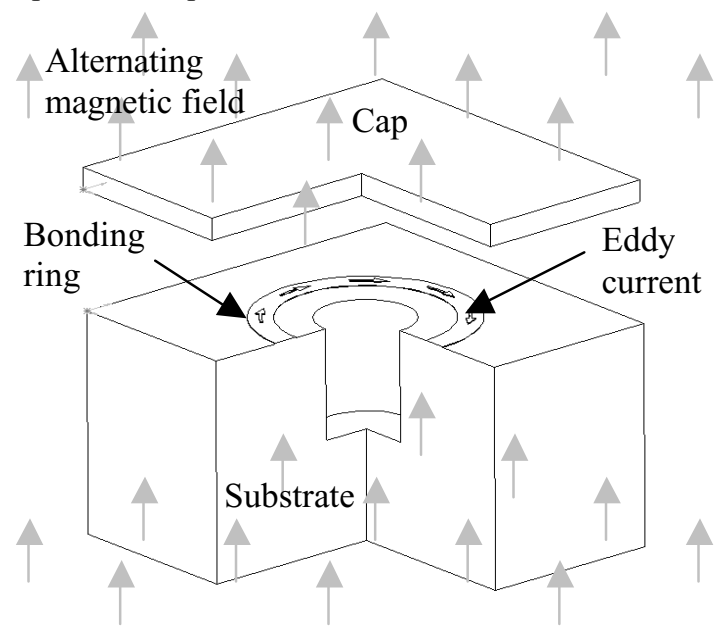

Figure 1. Induction heating and bonding concept. Using externally applied alternating magnetic field to induce eddy currents and resistive heating in a metallic bonding ring.

It is believed that modifications to the macroscopic induction heating theory was necessary, because some of the assumptions that were made while deriving those theorymight no longer hold in the microscopic scale. The gold bonding rings used is $6 \mu \mathrm{m}$ thick, has a $1 \mathrm{~mm}$ inner diameter and a width of 100 $200 \mu \mathrm{m}$. The electric field generated in the bonding ring is

$$
E=-\frac{d \Phi_{B}}{d t}=-K \mu A \frac{d I}{d t}
$$

Where $\mathrm{E}$ is the electromotive force (EMF) generated, $\Phi_{\mathrm{B}}$ is the magnetic flux, $\mu$ is the permeability, $\mathrm{A}$ is the cross sectional area, I is the current powering the coil, and $\mathrm{K}$ is the coil constant, which governs the strength of the magnetic field created for a given coil current. Given the small cross sectional area of the bonding rings, it is very difficult to generate a high EMF. As Eq. 1 implies, adequate heating requires a large high frequency current running through a coil that could generate a strong magnetic field for the bonding ring.

The induction bonding setup is shown in Fig. 2. The bonding sample is placed in the center of an 8 turned helical coil, which has a $12 \mathrm{~mm}$ inside diameter, $18 \mathrm{~mm}$ outer diameter, $3 \mathrm{~mm}$ tube diameter and a $5 \mathrm{~mm}$ gap between the top and bottom four turns. 


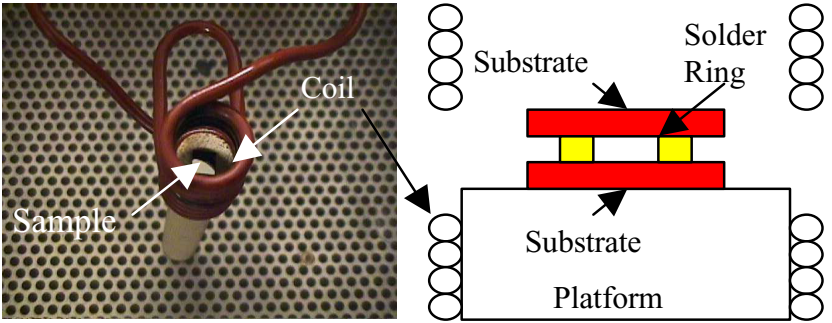

Figure 2. Induction heating setup using an 8 turned helical coil driven between $10-15 \mathrm{MHz}$.

The magnetic intensity applied to the bonding ring could be calculated by modeling the coil as 8 current loops each with a $7.5 \mathrm{~mm}$ radius and an axial distance $\mathrm{z}$ away from the bonding ring. The magnetic intensity contribution of each loop can be calculated using Eq. 2, which is derived from Ampere's law [10].

$$
H(z)=\frac{r^{2} I}{2\left(r^{2}+z^{2}\right)^{3 / 2}}
$$

Where I stand for the current running through the coil. With the coil geometry above, the magnetic intensity generated is approximately $150 \mathrm{I}(\mathrm{A} / \mathrm{m})$. Up to $180 \mathrm{Amps}$ can be generated in the coil, creating a magnetic intensity as high as $27,000(\mathrm{~A} / \mathrm{m})$ in our current setup.

During an induction heating operation, the coil and the heated work piece, e.g. a metal cylinder, are magnetically linked. The magnetic field generated by the coil induces an EMF and eddy current in the cylinder. As the current flows in the cylinder, it too creates a magnetic field. According to Lenz's law, the field the cylinder generates is opposite to the original magnetic field that was generating the eddy current. Hence as the incoming magnetic field travel towards the center of the cylinder, it is cancelled by the opposing magnetic field the induced eddy current generates. Therefore, less and less eddy current can be induced radially towards the center of the cylinder. The depth where the eddy current density reaches $1 / \mathrm{e}$ of the surface density is called the skin depth, defined as

$$
\delta=\sqrt{\frac{\rho}{f \pi \mu}}
$$

Where $\delta$ is the skin depth, $\rho$ is resistivity, and $f$ is the frequency. The above macroscopic induction heating theory [11-13] holds when the induced eddy current is large and the opposing magnetic field generated is sufficient to cancel the incoming magnetic field. In this case, most of the eddy current travels circumferentially in the first radial skin depth and generate resistive heating there. However, when the bonding ring is very small and thin, the induced voltage is low, and the resistance to eddy current flow is high. In this case, the induced eddy current and the opposing magnetic field created is low, therefore, the incoming magnetic field can penetrate deeper into the radial direction, inducing more eddy currents and more heating.

\section{EXPERIMENTAL RESULTS}

The prediction of induction heating modified for small thin bonding rings was tested using several different metal rings patterned on Pyrex substrates. Temperature indicating paint was used to determine the location of heat generation, as well as the temperature reached by the rings. Once the paint reaches its indicating temperature, it turns from opaque to clear; this process can take less than $10 \mathrm{~ms}$.

Several experiments confirmed that heat could be generated beyond the radial skin depth as defined by Eq. 3. Figure 3 shows pictures of concentric tungsten rings covered in $150^{\circ} \mathrm{C}$ temperature indicating paint heated under induction power.

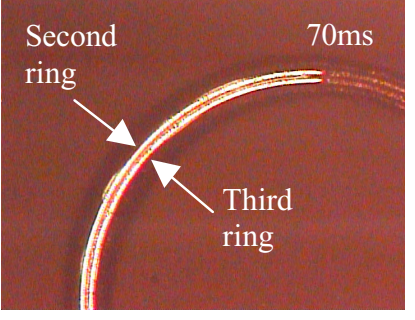

(a)

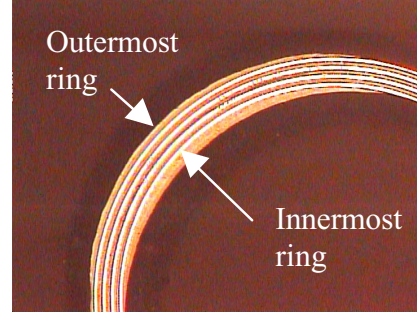

(b)
Figure 3. Tungsten bonding rings painted with $150^{\circ} \mathrm{C}$ temperature indicating paint and heated under induction power

The four rings are approximately $2 \mathrm{~mm}$ in diameter, $2 \mu \mathrm{m}$ thick, $20 \mu \mathrm{m}$ wide with a $20 \mu \mathrm{m}$ gaps between each ring. At $150^{\circ} \mathrm{C}$, the radial skin depth of tungsten calculated using Eq 3. is $40 \mu \mathrm{m}$. If the macroscopic skin depth theory holds, the outermost ring should produced the most heat, the second ring that is $40 \mu \mathrm{m}$ away from the outermost skin should have little resistive heating, and the innermost ring should have virtually no heating at all. Judging by Fig. 3, the paint started to melt from the center rings and spread equally to both sides of the innermost and outermost rings. It can be concluded that approximately equal heating originated from all of the rings. The center two rings heated up first because it was harder for the heat generated in those rings to be conducted away.

Heat generation between $6 \mu \mathrm{m}$ thick, $1 \mathrm{~mm}$ diameter gold rings with widths of $200 \mu \mathrm{m}$ and $50 \mu \mathrm{m}$ were compared. Induction power was applied to the gold ring until they started glowing at roughly $900^{\circ} \mathrm{C}$. The calculated skin depth of gold at this temperature is $45 \mu \mathrm{m}$. It was observed that $200 \mu \mathrm{m}$ wide rings required $400 \mathrm{~W}$ of coil power to sustain glowing while the $50 \mu \mathrm{m}$ ring required $1000 \mathrm{~W}$. Therefore, heat must be generated beyond the $45 \mu \mathrm{m}$ radial skin depth, otherwise the extra width of the gold ring would act as a heat sink to dissipate heat thus it should require more coil power to attain glowing. The fact that heat can be generated beyond the theoretical radial skin depth is important because more heating power can be couple into the metal rings than predicted by macroscopic theory [11-13]. It should be noted that at this small scale, metal rings with lower resistance, either made thicker, wider or made from more conductive metals tends to generate more heat. Figure 4 shows the temperature reached by a gold bonding ring with varying power level and heating time. When the coil power is at $100 \mathrm{~W}$, the temperature of the metal ring can only reach $400^{\circ} \mathrm{C}$ in 0.5 second. When the coil power is above $500 \mathrm{~W}$, the ring temperature reaches more than $1000^{\circ} \mathrm{C}$ in 0.5 second. However, increasing the coil power above $500 \mathrm{~W}$ show less dramatic increase in the temperature-time plot.

Temperature vs. Heating Time

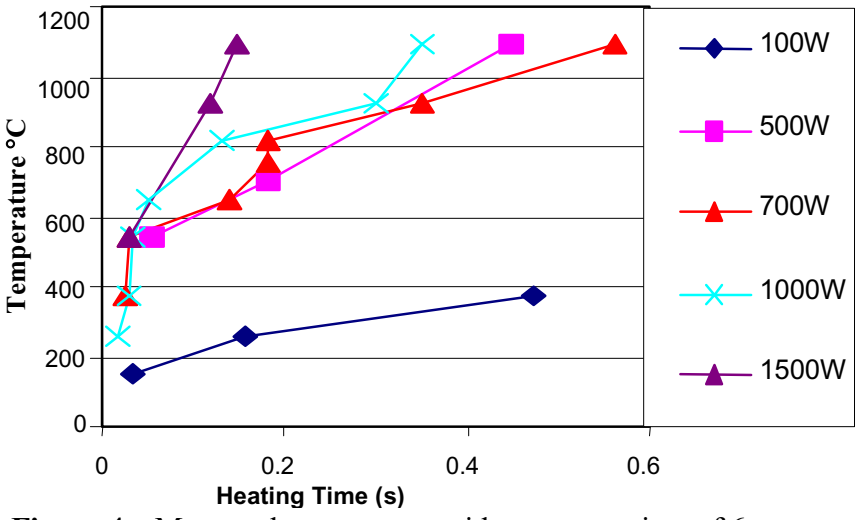

Figure 4. Measured temperature with respect to time of $6 \mu \mathrm{m}$ thick, $200 \mu \mathrm{m}$-wide and $1 \mathrm{~mm}$ diameter gold bonding ring under various induction heating power. 
Polycarbonate substrate has been bonded to glass substrate using gold bonding rings. The time needed to achieve bonding is $100 \mathrm{~ms}$. The adhesion strength of these bonds is limited by the adhesion strength of the gold bonding ring to the glass substrate. Forcefully breaking one of these bonds usually result in the gold bonding ring being peeled off from the glass substrate while adhering to the polycarbonate substrate. Due to the low thermal budget requirements of the bonding cycle, it is possible to encapsulate water using this bonding setup. Figure 5 shows picture of a polycarbonate cavity bonded with a glass cap encapsulating liquid water. The gold bonding ring is $1 \mathrm{~mm}$ across, $100 \mu \mathrm{m}$ wide, and $6 \mu \mathrm{m}$ thick. Since the bonding process only takes $100 \mathrm{~ms}$ and the thermal budget is low, the water droplet was expected to stay very close to its initial ambient temperature.

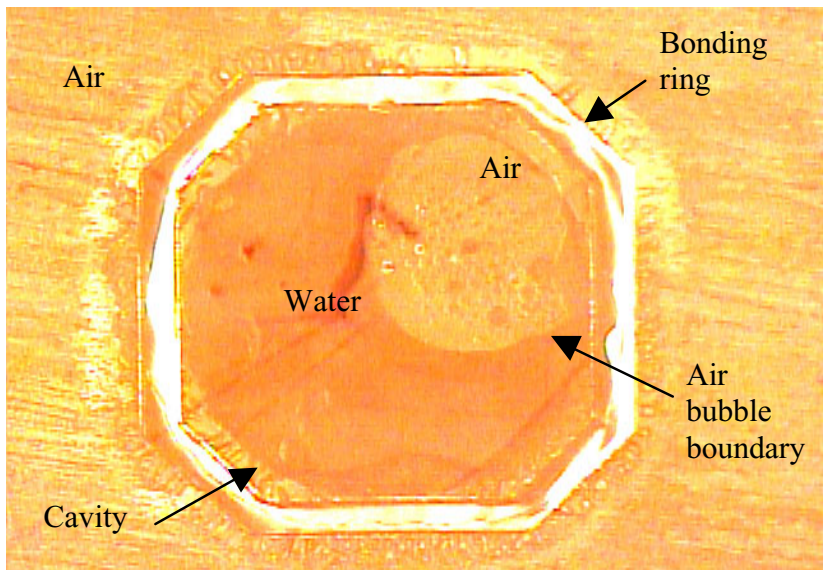

Figure 5. Water encapsulated between a cavity drilled in polycarbonate substrate and a glass cover plate

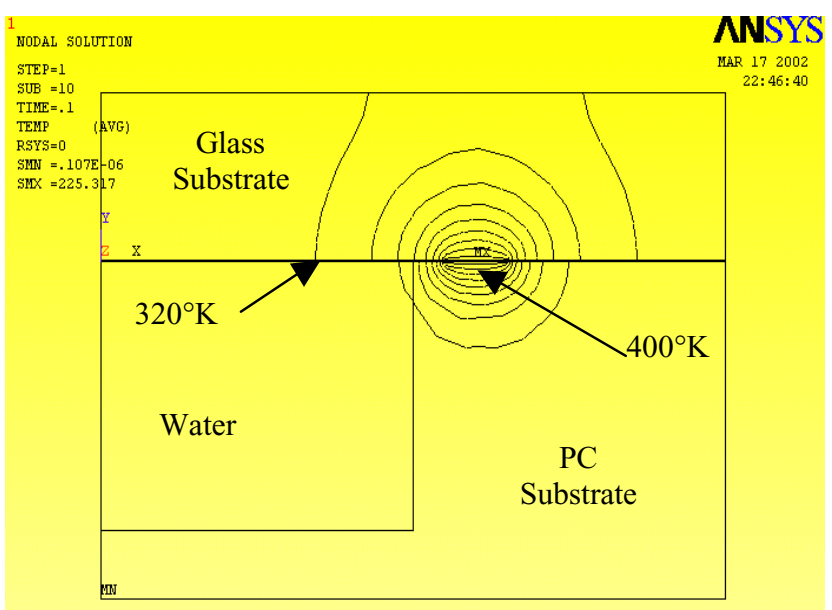

Figure 6. Finite element simulation of $100 \mathrm{~ms}$ Pyrex to Polycarbonate (PC) bond. Water encapsulated in the cavity will receive minimal heating

Figure 6 show a finite element analysis of the glass to polycarbonate bond at $100 \mathrm{~ms}$, which indicates the heat which originates from the bonding ring will not warm the water originally at $300^{\circ} \mathrm{K}$, encapsulated in the polycarbonate cavity. This is an important requirement for encapsulating bio-fluids, which can be damaged just few degrees above body temperature. This process may have potential application in sealing or encapsulating drugs, chemicals or bio-medical materials.
Pyres-gold-Pyrex bonding was accomplished using gold bonding rings $1.2 \mathrm{~mm}$ across, $6 \mu \mathrm{m}$ thick and either $100 \mu \mathrm{m}$ or $200 \mu \mathrm{m}$ wide. The gold rings, which were patterned on a Pyrex substrate, were kept in contact with another bare Pyrex substrate. Bonding was achieved using $750 \mathrm{~W}$ of coil power, heating the ring to a bright red glow at approximately $900^{\circ} \mathrm{C}$ for 60 seconds.

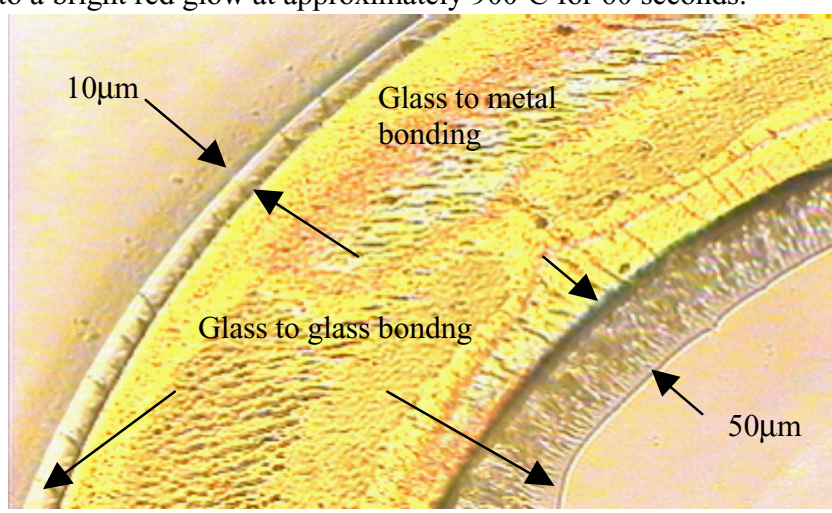

Figure 7. Pyrex-to-Pyrex bonding using $1 \mathrm{~mm}$ diameter, $200 \mu \mathrm{m}$ wide and $4 \mu \mathrm{m}$ thick gold solder.

Direct Pyrex-to-Pyrex bonding can be achieved by applying higher temperature and pressure to the glass-gold-glass bond system. The higher temperature softens the glass, and the applied pressure forces the molten glass to flow around the gold ring and bond to the molten glass on the opposing substrate. Figure 7 show a direct Pyrex-to-Pyrex bond on both the inner and outer boundaries of the gold bonding ring. This bond was achieved in 3 seconds using $1500 \mathrm{~W}$ of coil power.

Both the Pyres-gold-Pyrex, and direct Pyres-to-Pyrex bonds were very strong, they are comparable to the bulk strength of Pyrex substrate. Forcefully breaking one of these bonds usually resulted in tearing of the bulk substrate as shown in Fig. 8.

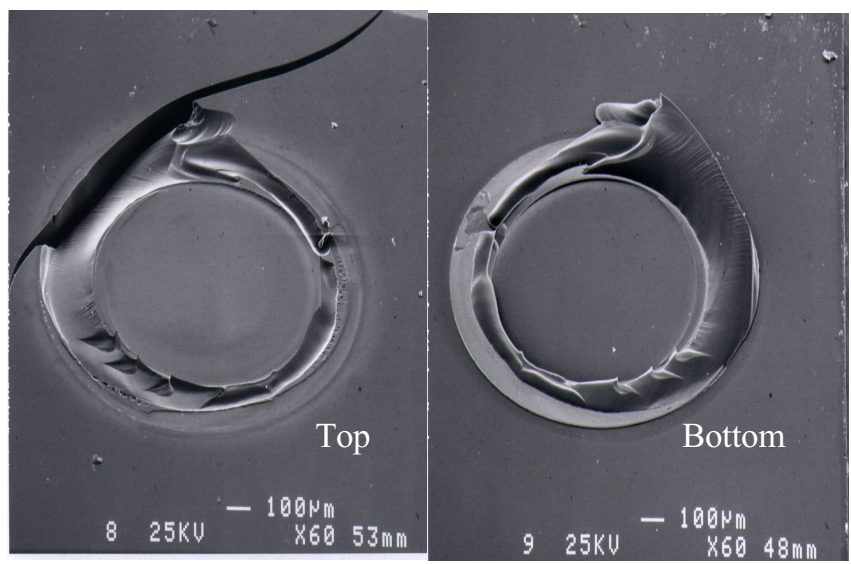

Figure 8. Two halves of a forcefully broken Pyrex-to-Pyrex bond.

Both types of bonds were tested for hermetic sealing using dyed isopropyl alcohol. Alcohol has a very low surface tension hence it can easily seep into a non-hermetic seal. The bond interface was flooded with alcohol for approximately 1 minute before the alcohol starts to evaporate away. Figure 9 show a Pyrex-goldPyrex bond that was once submerged in alcohol, now approximately half the alcohol evaporated away from the interface. The bonds were also tested in a $100 \%$ humidity, 2 atmosphere autoclave chamber for 30 minutes. Both kinds of bonds were able to keep moisture out of the enclosed cavity. 


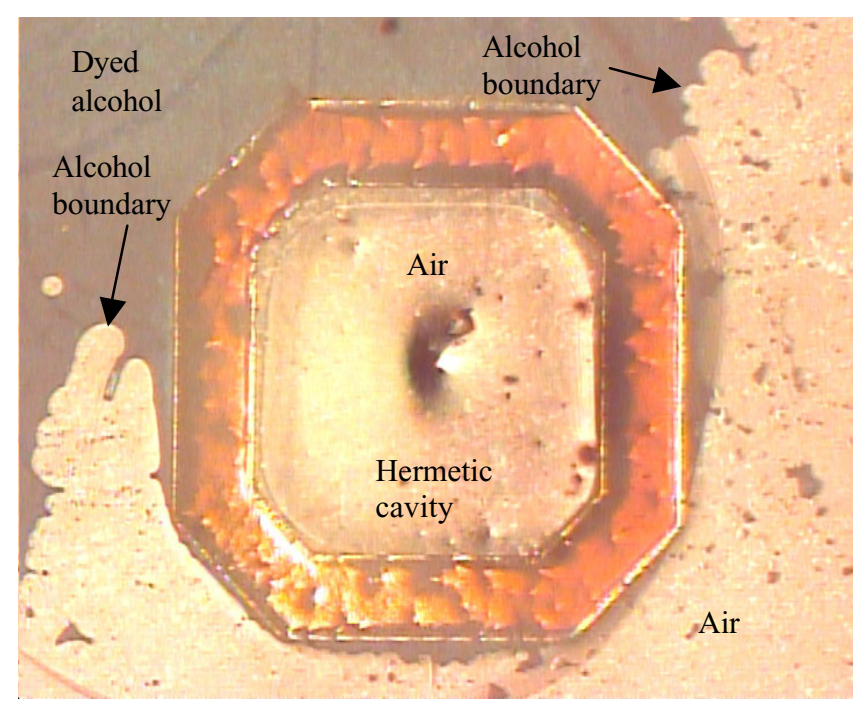

Figure 9. Alcohol cannot seep into the Pyrex-gold-Pyrex bonded cavity.

It is highly desirable to reduce the bonding time. Short heating cycles reduces the heat-affected zone, decreases the amount of energy needed for bonding, and increases production rate. Using induction heating, it is theoretically possible to achieve bonding very quickly. However short heating cycles like the 3 second Pyrex-to Pyrex bond mentioned earlier often suffer from localized burnouts. These failures are the result of uneven heating of the metal bonding rings. The electrical resistivity of most metals increases quickly with increasing temperature. Since the same amount of eddy current has to travels around the bonding ring with a constant cross sectional area; once a hot spot develops, the resistivity will locally increase, causing even more heat generation than its neighboring area. This effect can quickly lead to localized melting, vaporization, or oxidation of the bonding ring.

There are three methods to reduce the occurrence of burnouts. 1) Increase the heating power gradually. This allows the heat to conduct from the hot spots to its neighboring region, hence evening out the heat generated. 2) Use thicker bonding rings, hence process variations would not be as noticeable. 3) perform the bonding in an inert environment, this prevent oxidation of the heated metal ring. 4) Use metal that has a high melting temperature and is relatively inert. This is the reason why gold was chosen to make high temperature glass to glass bonding rings. Even though gold does not easily oxidize, hot spots can cause localized melting, and surface tension wicks the molten gold to the solid gold region and break the current loop. This effect can be seen in Fig 10.
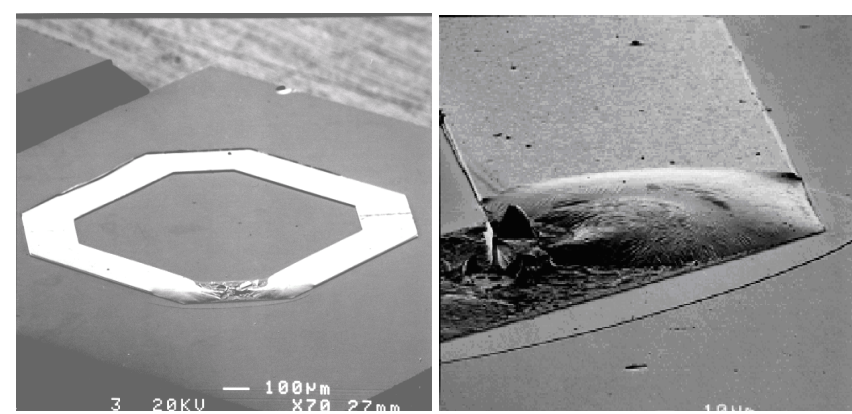

Figure 10. Localized melting of gold bonding ring a) one corner of the bonding has melted and broke the current loop. b) detailed view of melted region shows the melted region has a thicker and rounder cross sectional area than the original loop.

\section{CONCLUSION}

A modified theory of induction heating for MEMS scaled parts has been proposed and verified. It has been shown that for small thin parts, eddy current heating can be generated beyond the theoretical radial skin depth derived for macroscopic induction heating. In the micro-scale, metal rings with less resistance; either made wider, thicker, and of more conductive material can generate more heating.

Induction heating has been applied to perform strong, hermetic bonding in the microscale. The bonding temperature is approximately $900^{\circ} \mathrm{C}$ or above, and the bonding time ranged from 3 second to 60 seconds. Slow bonding cycles achieves hermetic bond on a more controllable and consistent basis. Rapid hermetic bonding is more prone to burnouts hence it has lower success rate. Future research will focus on sub-second induction bonding and encapsulation of MEMS devices

\section{ACKNOWLEDGEMENT}

These devices were fabricated in the UC Berkeley microfabrication laboratory. This work is supported in part by an NSF Career award (ECS-00960098) and a DARPA/MTO/MEMS grant

\section{REFERENCES}

[1] Liwei Lin, K. McNair, R.T. Howe and A.P. Pisano, "Vacuum Encapsulated Lateral Microresonators", $7^{\text {th }}$ Int. Conference on Solid State Sensors and Actuators, pp 170-273, Yokohama, Japan, June, 1993.

[2] Liwei Lin, "selective Encapsulation of MEMS: Micro Channels, Needles, Resonators, and Electromechanical Filters", Ph. D. Theses, UC Berkeley, 21, 1993

[3] Y.T Cheng, Liwei Lin, and Kahlil Najafi, "Localized Bonding with PSG or Indium Solder as intermediate Layer," Proceedings of IEEE Micro Electro Mechanical Systems Workshop, pp. 285-289, Orlando, Florida, 1999

[4] Y. T. Cheng, L. Lin, K. Najafi, "localized silicon fusion and Eutectic Bonding for MEMS Fabrication and Packaging", Technical Digest of the 1998 Solid State Sensor and Actuator Workshop, Hilton Head, pp 233-236, 1998

[5] Y. T. Cheng, W. T. Hsu, L. Lin, C. T. Nguyen, and K. Najafi. "Vacuum Packaging Technology Using Localized Aluminum/Silicon-to Glass Bonding", Technical Digest of the 2001 Micro Electro Mechanical System. Interlaken, Switzerland, pp18-21.

[6] Mu Chiao and Liwei Lin, "Hermetic Wafer Bonding Based On Rapid Thermal Processing. Solid State Sensor and Actuator Workshop. Hilton head, pp 347-350

[7] M. Chiao and Liwei Lin, "A Wafer-Level Vacuum Packaging Process by RTP Aluminum-to-Nitride Bonding”, to appear in this workshop.

[8] Thompson K. , Gianchandani Y. B, Booske J., Cooper R. SiSi Bonding Using RF and Microwave Radiation. The $11^{\text {th }}$ International Conference on Solid-State Sensors and Actuators, Munich, Germany, June 10-14, 2001. p226-229

[9] Andrew Cao and Liwei Lin "Selective Induction Heating for MEMS Packaging and Fabrication”. Proceedings of 2001 ASME International Mechanical Engineering Congress and Exposition, Nov 11-16, 2001, New York, NY

[10] Lieu, D. U.C Berkeley, ME 219 reader. Spring 2001

[11] Davies, J. Simpson. P. "Induction Heating Handbook". McGraw-Hill Book Company (UK) limited. 1926

[12] Zinn, S. and Semiatin S. L. Elements of Induction heating. Carnes Publication Services, Inc. 1991

[13] Curits, F. W. High-frequency Induction heating, second edition. McGraw Hill Book Company, Inc. 1950 